\title{
A Single-Stage Dimmable Multi-String HB-LED Driver with High Power Factor and Temperature Compensation
}

\author{
Y.H. Chiu, Y.H. Liu \\ National Taiwan University of Science and Technology \\ Taipei City, Taiwan
}

\author{
S.C. Wang \\ Lunghwa University of Science and Technology \\ Taoyuan City, Taiwan
}

\author{
Yuan-Lin Chen \\ Mingchi University of Technology \\ New Taipei City, Taiwan
}

\begin{abstract}
This paper proposes a single-stage constant-current driver with power factor correction (PFC) and temperature rise compensation for igniting multi-string high-brightness light-emitting diodes (HB-LEDs) in parallel connection. The devised dimmable LED driver is composed of a coupled inductor single-ended primary inductance converter (SEPIC) with PFC mechanism and k-channel current regulators (CRs) with dimming and temperature compensation capabilities. The LED voltage drop features negative temperature coefficient characteristic and is subject to change depending on the forward current and the thermal resistivity of a given package. By regulating the LED feeding voltage continuously, a dynamic voltage regulation (DVR) control is addressed in this paper to automatically minimize the power dissipation of the CR caused by the voltage drop variation due to the LED junction temperature drift. With low implemented outlay, the studied topology of multi-string LED driver has good power conversion efficiency and low total harmonic distortion (THD). The control mechanisms and design considerations are analyzed and discussed. An $80 \mathrm{~W}$ laboratory prototype implemented with two low-cost commercial controllers is also built and tested. Experimental results are provided to show the effectiveness and performance of the proposed scheme.
\end{abstract}

\section{Keywords- PFC; HB-LEDs; SEPIC; DVR}

\section{INTRODUCTION}

In recent years, the advances in high-brightness light-emitting diode (LED) technologies have made it as an attractive alternative to supersede the conventional lighting sources. Among the solid-state lighting (SSL) applications, the LED technology is environmentally friendly and free of toxic materials and infrared as well as ultraviolet radiation, in consequence they are becoming more and more popular due to the desirable characteristics such as high luminous efficiency, compact size, long lifetime, energy saving, wide color gamut, reliability, and fast response [1]-[4]. All these features make LEDs a good candidate to replace commonly used incandescent bulbs and discharge lamps in many lighting applications, such as street lighting, automotive lighting, liquid-crystal-display (LCD) backlighting, traffic lights, decorative applications, and household and emergent appliances etc. [5]-[10].

Since LED is a current-driven device and its brightness is related to the forward conduction current. Currently, the LED lighting systems powered by ac mains needs a converter with high power factor, low crest factor (CF) and low total harmonic distortion (THD) to meet the international standard IEC61000-3-2 Class C [9]-[13]. Power quality problems come from the ac line current distortion and, often occur in the conventional LED driver which has a front stage of the bridge rectifier with a bulky electrolytic capacitor. Hence, the PFC pre-regulator is required to shape the line current profile to maximize the power factor, while the LED driver provides the current regulation and the dimming control. For driving multiple LED strings, multiple constant-current regulators, which are composed of MOSFETs with current sensing resistors, are used in series with individual LED strings to maintain the current passing through the LED string [14], [15]. On the other hand, LED drivers with an adjustable drive voltage were proposed in [16], [17]. In [16], [17], the maximum gate voltage of the MOSFETs in the current controllers is detected by sensing diodes to adaptively regulate the applied LED voltage to maintain the minimum voltage drop across the MOSFETs of the current controllers.

In this paper, a single-stage high-efficacy SEPIC PFC with LED feeding voltage dynamic regulation control to compensate the change of the LED VFD induced by the junction temperature drift for minimizing power losses produced in current regulator circuit is designed and realized for the LED driver. The drain voltage of the MOSFET switch in $\mathrm{CR}$ in individual LED string is detected when the LED working temperature has changes. The minimum drain voltage is determined through a simple logical deduction unit. Then, a desired margin voltage corresponding to the sensed minimum drain voltage is figured out and it is added to the primary feedback loop of the SEPIC converter to regulate the applied LED string voltage dynamically. Thus, even the voltage drops across the LEDs are changed due to the heat; the proposed scheme can make a suitable decision and finely tune the 
converter output voltage to minimize the voltage drop across the MOSFET and current sensed resistor in the CR for individual LED string. With low design and realization cost, the studied multi-string LED driver has good power conversion efficiency, high power factor, and low THD completely complied with the IEC61000-3-2 Class C standard.

\section{SYSTEM CONFIGURATION OF LED DRIVER}

Fig. 1 shows the system configuration of the proposed LED driver. From Fig. 1, the LED driver mainly consists of a SEPIC PFC controlled by the integrated circuit 2 (IC 2), constant current regulators, a dimming control circuit, a dynamic voltage regulator (DVR) controlled by the IC 1, and k-channel LED strings under drive.

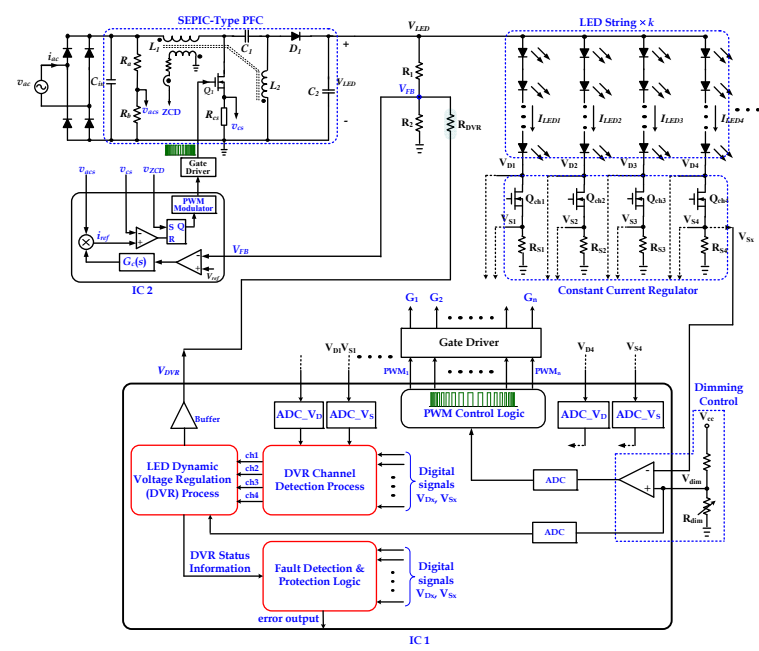

FIGURE I. FRAMEWORK OF THE PROPOSED LED DRIVER WITH DVR CONTROL.

\section{A. Coupled Inductor SEPIC PFC Based on Critical Conduction Mode (CRM)}

Fig. 2 illustrates the studied coupled inductor SEPIC PFC scheme. The line voltage, main switch current, zero current detection, and output voltage are sensed. The sampling signals are compared to the references and compensated in the PFC IC to form closed-loop control and complete the CRM operation. Form Fig. 2, if the bridge rectifier is ideal and the line voltage is purely sinusoidal, then the PFC input voltage vin can be expressed by

$$
v_{\text {in }}(t)=V_{m} \cdot|\sin (\omega t)|=\sqrt{2} V_{r m s} \cdot|\sin (\theta)|, \quad \theta=\omega t=2 \pi f t
$$

The peak current ipk of the main switch will follow the sinusoidal voltage reference and can be denoted by

$$
i_{p k}(\theta)=I_{p k} \cdot|\sin (\theta)|
$$

In CRM operation, the Q1 current is the summation of iL1 plus iL2 when the Q1 is on. Thus, the ipk can be obtained as

$i_{p k}(\theta)=i_{L 1}(\theta)+i_{L 2}(\theta)=I_{L 10}+\frac{v_{\text {in }}(\theta)}{L_{1}} \cdot t+I_{L 20}+\frac{V_{\text {in }}(\theta)}{L_{2}} \cdot t=\left(\frac{1}{L_{1}}+\frac{1}{L_{2}}\right) \cdot t_{o n}(\theta) \cdot\left(\sqrt{2} V_{\text {ms }}|\sin (\theta)|\right)$
Where the summation of the initial currents $I_{L 10}$ and $I_{L 20}$ equals to zero. Fig. 3 shows the waveforms of the iQ1 and iD1 and their peak sinusoidal envelope.

From (3), the conduction time ton can be derived as

$$
t_{o n}(\theta)=\frac{I_{p k}|\sin (\theta)|}{\left(\frac{1}{L_{1}}+\frac{1}{L_{2}}\right)\left(\sqrt{2} V_{r m s}|\sin (\theta)|\right)}=\frac{I_{p k} L_{e}}{\sqrt{2} V_{r m s}}=t_{o n}
$$

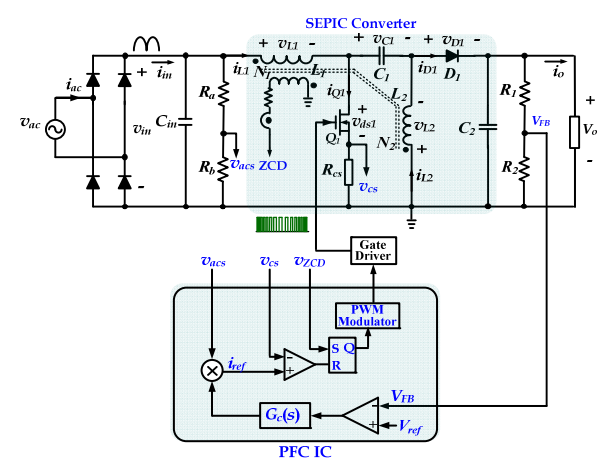

FIGURE II. THE P-V CURVE OF THE PHOTOVOLTAIC MODULE.

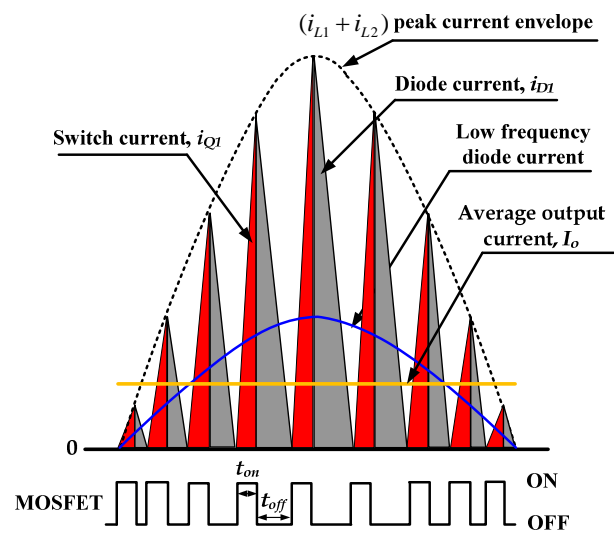

FIGURE III. WAVEFORMS OF THE IQ1 AND ID1 AND THEIR PEAK SINUSOIDAL ENVELOPE.

Where $L_{e} \triangleq L_{1} \| L_{2}$. From (4), the switch on time is independent of $\theta$. On the other hand, with the volt-second balance of the L2, the switch off time can be obtained as

$$
t_{\text {off }}(\theta)=\frac{V_{\text {in }}(\theta) t_{\text {on }}}{V_{o}}=\frac{t_{\text {on }} \sqrt{2} V_{\text {rms }}|\sin (\theta)|}{V_{o}}
$$

Consequently, the switching frequency can be obtained as

$$
f_{s}(\theta)=\frac{1}{t_{o n}+t_{\text {off }}(\theta)}=\frac{1}{t_{o n} \cdot\left(1+\frac{\sqrt{2} V_{r m s}|\sin (\theta)|}{V_{o}}\right)}
$$


According to the input average power and the design specification (output power Po, efficiency $\eta$, and input voltage Vrms), the peak input current can be computed by

$$
I_{p k}=\frac{2 P_{o}}{\eta \cdot \sqrt{2} V_{r m s} \cdot F\left(k_{v}\right)}
$$

Where

and $\quad \frac{1}{T} \int_{0} \overline{1+k_{v}|\sin (\omega t)|}$. From (4), (5), and (7), the switching frequency can be rewritten as

$$
f_{s}(\theta)=\frac{\eta V_{r m s}^{2} F\left(k_{v}\right)}{P_{o} L_{e}\left(1+k_{v}|\sin (\theta)|\right)}
$$

The minimum switching frequency (fs-min) occurs in the lowest input voltage Vrms-min and $\sin (\theta)=1$, and can be expressed by

$$
f_{s-\min }=\frac{\eta V_{r m s-\min }^{2} F\left(k_{v-\min }\right)}{P_{o} L_{e}\left(1+k_{v-\min }\right)}
$$

Where

$$
\begin{gathered}
k_{v-\min } \equiv \sqrt{2} V_{r m s-\min } / V_{o} \text { and } F\left(k_{v-\min }\right) \equiv \frac{1}{T} \int_{0}^{T} \frac{\sin ^{2}(\omega t)}{1+k_{v-\min }|\sin (\omega t)|} d t \\
\text { III. CONTROL MECHANISM OF DYNAMIC VOLTAGE } \\
\text { REGULATION }
\end{gathered}
$$

\section{Control Mechanism of Dynamic Voltage}

Fig. 4 illustrates the control loops of the presented dynamic voltage regulation (DVR) method. From Fig. 4, two feedback control loops, i.e. primary loop and secondary loop, are used to control the SEPIC converter output voltage (i.e. the VLED). The primary loop is the original control loop within the SEPIC used to accurately control the PFC output voltage. The output voltage VLED is sensed by a voltage divider which is comprised of precision resistors. The sensed signal VFB is compared with a reference Vref via an error amplifier inside the PFC IC. The PID compensator is utilized to provide an adequate gain compensation for improving system steady-state error and to supply a suitable phase margin to stabilize the system and optimize system transient response. On the other hand, the secondary loop is the dynamic voltage regulation loop used to finely adjust the LED string supply voltage for compensating the changes of the LED forward voltage drop due to the temperature variation. According to the sensed VD voltages of the MOSFETs in all current control circuits, via the logic derivation and computation of the DVR deduction logic unit, an optimum DVR voltage VDVR is determined and outputted through a buffer. The VDVR is added to the original feedback control loop (primary loop) of the SEPIC PFC to produce a minimum supply voltage for all parallel LED strings provided that the required LED dimming currents are met.

From Fig. 4, the VLED can be derived as

$$
V_{L E D}=\frac{V_{r e f}-V_{D V R}}{R_{D V R}} \cdot R_{1}+V_{r e f}\left(1+\frac{R_{1}}{R_{2}}\right)
$$

Where $\mathrm{V}_{\text {ref }}$ is the reference voltage of the SEPIC feedback control loop (primary loop), VDVR is the output of the dynamic voltage regulator. In this paper, the range of the regulable dynamic voltage is specified as VLED $\pm 20 \%$.

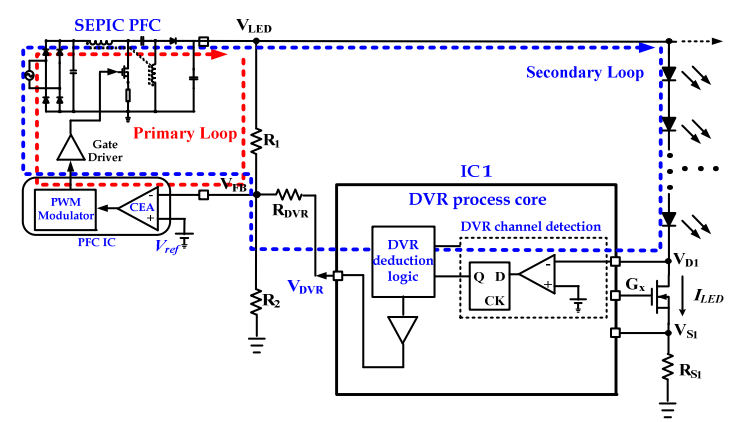

FIGURE IV. CONTROL LOOP OF THE DYNAMIC VOLTAGE REGULATION

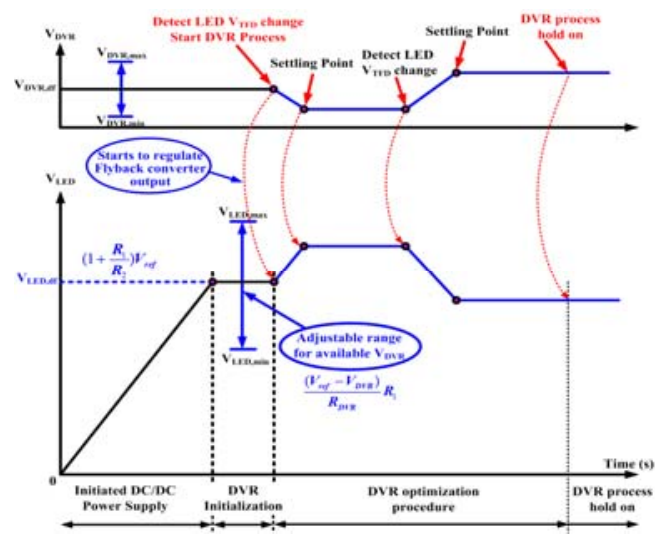

FIGURE V. THE FLOW CHART OF THE TRADITIONAL P\&O ALGORITHM

Fig. 5 shows the control mechanism of the proposed DVR method for the LED strings. From Fig. 5, when the system powers on, the initial VDVR is identical to the defaulted value VDVR,df. From (10), the corresponding output of the SEPIC PFC equals the preset output voltage VLED,df $=$ Vref $(1+\mathrm{R} 1 / \mathrm{R} 2)$. As the DVR processor detects the LED VTFD variation due to the temperature change, the procedure of the VLED automatic regulation is started in. If the VTFD is decreased (i.e. temperature rising), then the sensed VD is increased. At this time, the DVR control unit will increase the VDVR. From (10), when the VDVR increases, the VLED will decrease accordingly to reduce the voltage across the MOSFET and current sense resistor, then the power loss dissipated on the current controller will diminish for efficiency improvement. On the other hand, if the VTFD increases (i.e. temperature falling), then the sensed VD decreases. At this time, the DVR control unit will decrease the VDVR. From (10), when the VDVR decreases, the VLED will increase accordingly to reduce the power loss. If the VDVR holds on constant, it means that the VTFD has no variation, and then the 
DVR process unit will maintain the former operating state. The DVR procedure also memorizes the obtained best LED string supply voltages for the next operation use.

\section{EXPERIMENTAL RESULTS}

The specification of the single LED under test is $1 \mathrm{~W}$, $3.3 \mathrm{~V} / 250 \mathrm{~mA}$. The connection way is twelve LEDs in a series and eight series in parallel. Table 1 illustrates the design specification of the SEPIC PFC. The L6562 from ST Microelectronics Co. is used to implement the single stage SEPIC PFC under CRM control. The functionalities of the dynamic voltage regulation, constant current drives, and PWM dimming control are realized by the TLC5960 from Texas Instruments Co. Shown in Fig. 10 is the measured waveform of the ac line voltage and current at vac $=110 \mathrm{~V}$ and full load (80W). From Fig. 6, we can see that the line current is corrected to very approximate to the sinusoidal waveform and almost in phase with the line voltage. The power factor and efficiency versus various output powers is shown in Fig. 7. Fig. 8 shows the comparison between the different-order harmonic current and the IEC61000-3-2 Class $C$ regulation at full load $110 \mathrm{~V}$, we can see that the THD is restricted within the allowable tolerance and complies with the IEC 61000-3-2 Class C standard. Fig. 9 shows the photos of the realized LED driver prototype.

TABLE I. DESIGN SPECIFICATION OF THE SEPIC PFC

\begin{tabular}{|c|c|}
\hline Ac line voltage $\left(V_{a c, r m s}\right)$ & $90 \sim 130 \mathrm{~V}, 50 / 60 \mathrm{~Hz}$ \\
\hline Output voltage $\left(\mathrm{V}_{\mathrm{o}}\right)$ & $40 \mathrm{~V}$ \\
\hline Output current $\left(\mathrm{I}_{0}\right)$ & $2 \mathrm{~A}$ \\
\hline Output power $\left(\mathrm{P}_{\mathrm{o}}\right)$ & $80 \mathrm{~W}$ \\
\hline $\begin{array}{l}\text { Minimum switching } \\
\text { frequency }\left(f_{s-\min }\right)\end{array}$ & $25 \mathrm{kHz}$ \\
\hline Maximum efficiency ( $\boldsymbol{\eta}$ ) & $>85 \%$ \\
\hline Power factor (PF) & $>0.95$ \\
\hline $\begin{array}{l}\text { Total harmonic distortion } \\
\text { (THD) }\end{array}$ & $\begin{array}{l}\text { Comply with } \\
\text { IEC61000-3-2 C } \\
\text { standard }\end{array}$ \\
\hline Output voltage ripple & $<5 \%$ \\
\hline
\end{tabular}

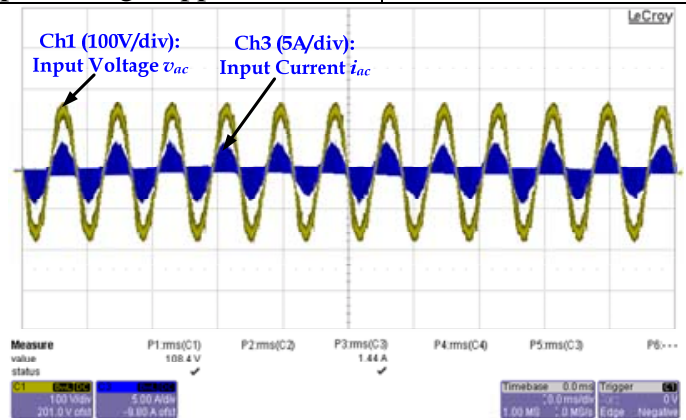

FIGURE VI. WAVEFORMS OF LINE VOLTAGE AND CURRENT.

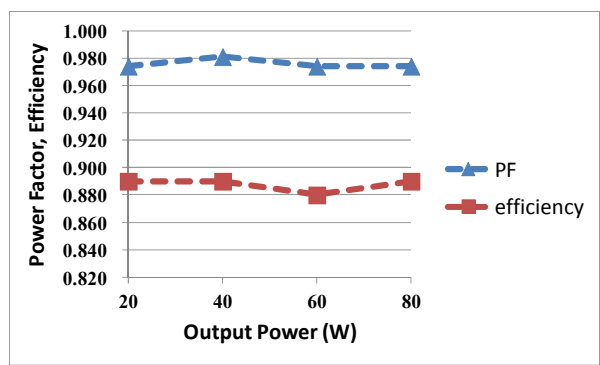

FIGURE VII. POWER FACTOR AND EFFICIENCY VS. OUTPUT POWER.

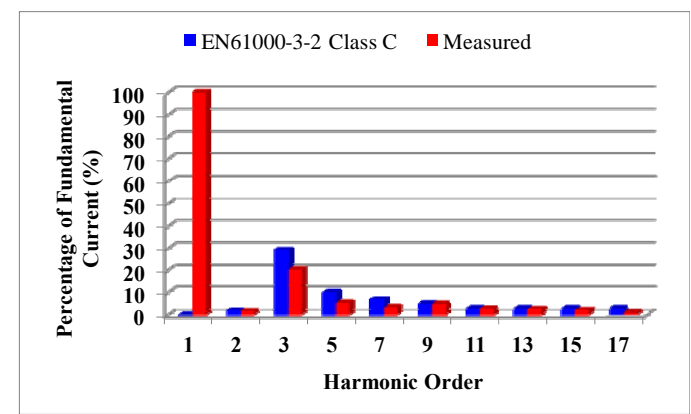

FIGURE VIII. FREQUENCY SPECTRA OF INPUT CURRENT.

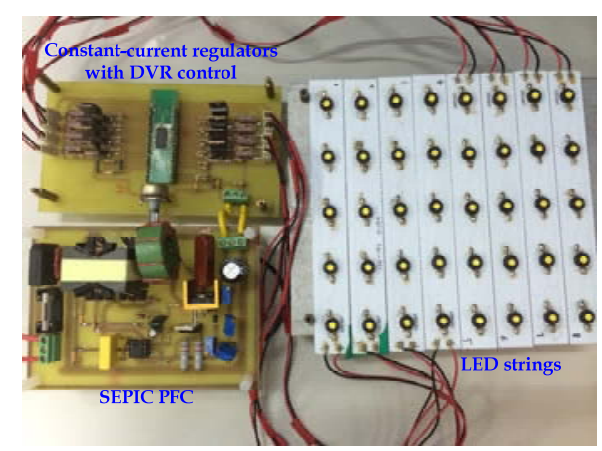

FIGURE IX. PHOTOS OF THE REALIZED LED DRIVER PROTOTYPE.

\section{CONCLUSION}

A single stage high power factor SEPIC converter with power factor correction for driving multi-string LEDs has been developed in this paper. The LED driver has merits of high power factor and low THD in low-cost outlay. In order to reduce the power dissipations in the constant current circuit, a DVR control is presented to adjust the LED supply voltage by means of the sense of the drain voltage of the MOSFET in the current regulator. The proposed DVR method features temperature compensation functionality and can improve the driver efficiency by minimizing the power losses of the constant current regulator. An 80W LED driver is designed and implemented; the experimental results verify the performance and effectiveness of the proposed control method and drive topology.

\section{ACKNOWLEDGMENT}

This work was supported financially in part by the National Science Council, Taiwan, under Grant NSC102-2622-E-262-003-CC3. 


\section{REFERENCES}

[1] C. Branas, F. J. Azcondo, and J. M. Osalonso, "Solid-state lighting: A system review,” IEEE Ind. Electron. Mag., pp. 6-14, Dec. 2013.

[2] Q. Luo, S. Zhi, C. Zou, W. Lu, and L. Zhou, "An LED driver with dynamic high-frequency sinusoidal bus voltage regulation for multistring applications,” IEEE Trans. Power Electron., vol. 29, no. 1, pp. 491-500, Jan. 2014.

[3] D. Park, Z. Liu, and H. Lee, “A $40 \mathrm{~V} 10 \mathrm{~W}$ 93\%-efficiency current-accuracy-enhanced dimmable LED driver with adaptive timing difference compensation for solid-state lighting applications," IEEE J. Solid-State Circuits, vol. 49, no. 8, pp. 1848-1860, Aug. 2014.

[4] M. S. Lin and C. L. Chen, "An integrated lighting unit with regulated pulse current driving technique," IEEE Trans. Ind. Electron., vol. 60, no. 10, pp. 4694-4701, Oct. 2013.

[5] C.A. Cheng, H.L. Cheng, and T.Y. Chung, "Novel single-stage high-power-factor LED street-lighting driver with coupled inductors," IEEE Trans. Ind. Appl., vol. 50, no. 5, pp. 3037-3045, Sept./Oct. 2014.

[6] L. Corradini and G. Spiazzi, "A high-frequency digitally controlled LED driver for automotive applications with fast dimming capabilities," IEEE Trans. Power Electron., vol. 29, no. 12, pp. 6648-6659, Dec. 2014.

[7] Y. L. Lin, H. J. Chiu, Y. K. Lo, and C. M. Leng, "LED backlight driver circuit with dual-mode dimming control and current-balancing design," IEEE Trans. Ind. Electron., vol. 61, no. 9, pp. 735-743, Sept. 2014.

[8] Y. H. Liu, Z. Z. Yang, and S. C. Wang, "A novel sequential-color RGB-LED backlight driving system with local dimming control and dynamic bus voltage regulation," IEEE Trans. Consumer Electron., vol. 56, no. 4, pp. 2445-2452, Nov. 2010.

[9] S.C. Moon, G.B. Koo, and G.W. Moon, "Dimming-feedback control method for TRIAC dimmable LED drivers," IEEE Trans. Ind. Electron., vol. 62, no. 2, pp. 960-965, Feb. 2015.

[10] R. A. Pinto, M. R. Cosetin, A. Campos, M. A. D. Costa, and R. N. do Prado, “Compact emergency lamp using power LEDs," IEEE Trans. Ind. Electron., vol. 59, no. 4, pp. 1728-1738, Apr. 2012.

[11] C.A. Cheng, C.H. Chang, T.Y. Chung, and F.L. Yang, "Design and implementation of a single-stage driver for supplying an LED street-lighting module with power factor corrections," IEEE Trans. Power Electron., vol. 30, no. 2, pp. 956-966, Feb. 2015.

[12] Y. Hu, L. Huber, and M. M. Jovanovic', "Single-stage, universal-input AC-DC LED driver with current-controlled variable PFC boost inductor," IEEE Trans. Power Electron., vol. 27, no. 3, pp. 1579-1588, Mar. 2012.

[13] H.J. Chiu, Y.K. Lo, J.T. Chen, S.J. Cheng, C.Y. Lin, and S.C. Mou, “A high-efficiency dimmable LED driver for low-power lighting applications," IEEE Trans. Ind. Electron., vol. 57, no. 2, pp. 735-743, Feb. 2010.

[14] M. Doshi and R. Zane, "Digital architecture for driving large LED arrays with dynamic bus voltage regulation and phase shifted PWM," in Proc. IEEE APEC, 2007, pp. 287-293.

[15] M. Doshi and R. Zane, "Control of solid-state lamps using a multiphase pulsewidth modulation technique," IEEE Trans. Power Electron., vol. 25, no. 7, pp. 1894-1904, Jul. 2010.

[16] Y. Hu and M. M. Jovanovic, "A novel LED driver with adaptive drive voltage," in Proc. IEEE APEC, 2008, pp. 565-571.

[17] Y. Hu and M. M. Jovanovic, "LED driver with self-adaptive drive voltage," IEEE Trans. Power Electron., vol. 23, no. 6, pp. 3116-3125, Nov. 2008. 